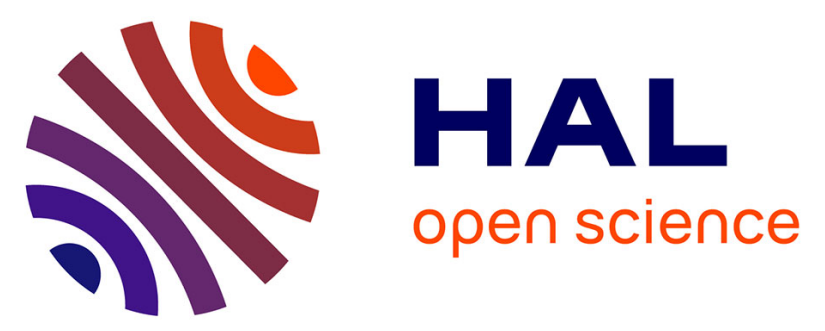

\title{
High-performance size exclusion chromatography with online fluorescence and multi-wavelength absorbance detection for isolation of high-purity carbon dots fractions, free of non-fluorescent material
}

Olga Trubetskaya, Oleg Trubetskoj, Alexey Vervald, Sergey Burikov, Victor Marchenkov, Olga Shenderova, Svetlana Patsaeva, Tatiana Dolenko, Claire Richard

\section{- To cite this version:}

Olga Trubetskaya, Oleg Trubetskoj, Alexey Vervald, Sergey Burikov, Victor Marchenkov, et al.. Highperformance size exclusion chromatography with online fluorescence and multi-wavelength absorbance detection for isolation of high-purity carbon dots fractions, free of non-fluorescent material. Journal of Chromatography A, 2021, 1650, pp.462251. 10.1016/j.chroma.2021.462251 . hal-03265724

HAL Id: hal-03265724

https://hal.science/hal-03265724

Submitted on 22 Jun 2021

HAL is a multi-disciplinary open access archive for the deposit and dissemination of scientific research documents, whether they are published or not. The documents may come from teaching and research institutions in France or abroad, or from public or private research centers.
L'archive ouverte pluridisciplinaire HAL, est destinée au dépôt et à la diffusion de documents scientifiques de niveau recherche, publiés ou non, émanant des établissements d'enseignement et de recherche français ou étrangers, des laboratoires publics ou privés. 
1 High-performance size exclusion chromatography with online fluorescence

\section{and multi-wavelength absorbance detection for isolation of high-purity} carbon dots fractions, free of non-fluorescent material

Olga E. Trubetskaya ${ }^{\mathrm{a}, *}$, Oleg A. Trubetskoj ${ }^{\mathrm{b}}$, Claire Richard $^{\mathrm{c}, \mathrm{d}}$, Alexey M. Vervald ${ }^{\mathrm{e}}$, Sergey A. Burikov ${ }^{\mathrm{e}}$, Victor V. Marchenkov ${ }^{\mathrm{f}}$, Olga A. Shenderova ${ }^{\mathrm{g}}$, Svetlana V. Patsaeva ${ }^{\mathrm{e}}$, Tatiana A. $\operatorname{Dolenko}^{\mathrm{e}, *}$

${ }^{\mathrm{a} B r a n c h}$ of Shemyakin and Ovchinnikov Institute of Bioorganic Chemistry, Russian Academy of Sciences, 6, Prospekt Nauki, 142290 Pushchino, Moscow region, Russia

${ }^{\mathrm{b}}$ Institute of Basic Biological Problems, Russian Academy of Sciences, 2, Institutskaya str., 142290 Pushchino, Moscow region, Russia

${ }^{c}$ Clermont Université, Université Blaise Pascal, Institut de Chimie de Clermont-Ferrand, Equipe Photochimie, BP 10448, F-63000 Clermont-Ferrand, France

${ }^{\mathrm{d}}$ CNRS, UMR 6296, ICCF, F-63171 Aubiere, France

${ }^{\mathrm{e}}$ Faculty of Physics, M.V. Lomonosov Moscow State University, 1, bldg.2, Leninskie Gory, 119991 Moscow, Russia

${ }^{\mathrm{f}}$ Institute of Protein Research, Russian Academy of Sciences, 4, Institutskaya str., 142290 Pushchino, Moscow region, Russia

${ }^{\mathrm{g}}$ Adamas Nanotechnologies, Inc., 8100 Brownleigh Dr, Suit 120, Raleigh, NC27617, USA

*corresponding authors.

E-mail addresses: olegi03@yahoo.com (O. E. Trubetskaya, Tel.: +7(4967)73-08-59\#3227), olegi03@rambler.ru (O. A. Trubetskoj), claire.richard@uca.fr (C. Richard), alexey.vervald@physics.msu.ru (A. M. Vervald), sergey.burikov@gmail.com (S. A. Burikov), vmarch@rambler.ru (V. V. Marchenkov), oshenderova@adamasnano.com (O. A. Shenderova), spatsaeva@mail.ru (S. V. Patsaeva), tdolenko@lid.phys.msu.ru (T. A. Dolenko, Tel.: +7(495)939-16-53; Fax: +7(495)939-11-04).

\section{ABSTRACT}

The carbon dots (CDs) from natural nanographite oxide mixture (NGO-MIX) and from its fraction NGO (3.5-10K) recovered after ultrafiltration and dialysis were analyzed by 
3D-excitation/emission matrix and high-performance size exclusion chromatography (HPSEC) combined with online fluorescence and absorbance detections. HPSEC chromatograms obtained simultaneously with absorption within the wavelength range 200$500 \mathrm{~nm}$ and fluorescence detection at $\lambda_{\mathrm{exc}} / \lambda_{\mathrm{em}}=270 / 450 \mathrm{~nm} / \mathrm{nm}$ showed that NGO-MIX sample is not homogeneous and consist of well resolved CDs fractions with different sizes, absorption spectra and distinct fluorescence and non-fluorescence properties. Despite the twice higher fluorescence intensity of fraction NGO (3.5-10K) compared to the NGO-MIX, some impurity of non-fluorescent components was detected by HPSEC. The absorbance spectra of chromatographic peaks, extracted from the data of multi-wavelength absorbance detector, demonstrated different combinations of absorbance maxima. It means that different chromatographic peaks correspond to sized and chemically different CDs fractions. This study demonstrated for the first time the possibility of separating oxidized nanographite into homogeneous free from non-fluorescent material CDs fractions with their simultaneous spectroscopic characterization.

KEYWORDS: natural nanographite oxide, carbon dots, absorbance, fluorescence, EEM, HPSEC

\section{Introduction}

At the present time much attention is devoted to carbon dots (CDs), which along with natural biocompatibility demonstrate stable intense fluorescence sensitive to changes in the environment [1]. These properties open up prospects for the utilization of these nanoparticles as optical sensors for various impurities in liquid and gaseous media [2-5], and as theranostic nanocomplexes that simultaneously act as luminescent biomarkers and drug carriers [6-9]. The relevance of using such CDs is primarily associated with the active development of science and technology, where the issue of controlling the content of various substances in multicomponent media, monitoring the occurrence of chemical reactions in various technological processes, and the need to create luminescent medical nano-agents are becoming increasingly acute [10].

The CDs can be synthesized by a variety of physicochemical methods from various carbon-based starting materials [11-14]. In most cases, the end product of CDs synthesis is a complex mixture of compounds with significantly different properties. Unfortunately, at 
present, there is no exact way to certify the compositon, which significantly complicates the development of a methodology for their application in specific tasks. The major drawback limiting the application of CDs in the life sciences is their not fully understood structure and relatively low quantum yield. Obtaining pure CDs, devoid of various non-fluorescent impurities, should help to reveal their natural chemical structure and provide important insights for understanding the optical properties of CDs synthesized by a strong oxidation process. The isolation of homogeneous fluorescent CDs free of non-fluorescent compounds will significantly increase the quantum yield, sensitivity, and, consequently, the operational efficiency of CD-based nanosensors.

Currently, a number of methods were suggested for CDs separation and purification including electrophoretic techniques, ultrafiltration and dialysis, solvent extraction, density gradient centrifugation or differential centrifugation, reverse phase, and anion exchange highperformance liquid chromatography [15-19]. Due to the fact that most as-prepared CD mixtures contain nanoparticles of different sizes, size-exclusion chromatography (SEC) becomes one of the most promising methods for the task. For this reason, various SEC methods with different combinations of solid and mobile phases including conventional lowpressure size-exclusion chromatography (conventional SEC) [20-25] and high-performance size-exclusion liquid chromatography (HPSEC) [26] have been used previously for purification and fractionation of CDs mixtures. However, detailed size-dependent fluorescence properties of CDs have not been clearly understood and sometimes controversial. Several research groups used preparative conventional SEC on the column with Sephadex G-100 in water as eluent for purification of fluorescent CDs from uncolored reagents in mixtures, prepared from carbon soot by Hammers method and reduced by $\mathrm{NaBH}_{4}$ [20], passivated by $\mathrm{PEG}_{1500 \mathrm{~N}}$ [21], doped by $\mathrm{ZnS}$ or $\mathrm{TiO}_{2}$ [22], without online detectors. The fluorescence emission and excitation spectra of CDs mixtures before and after purification were found to be rather similar, while their fluorescence quantum yields increased by several times [20-22]. The CDs mixture, extracted from instant coffee powder, were cleaned without online detectors from uncolored non-fluorescent compounds by conventional SEC on Sephadex G-25 in water and the mixture of CDs of different sizes has been obtained [23].

Arcudi et al. [24] divided the CDs mixture obtained from arginine and ethylenediamine by the microwave method into three fractions based on the elution volumes (Ve) using fractionation on Sephadex LH-20 in methanol as the eluent. However, the authors have shown neither the chromatographic column size nor the Ve of fractions. Therefore, it is not clear if 
fractions were eluted from the column due to the size exclusion effect or due to hydrophobic, ionic or other interactions with the solid phase. Kokorina et al. [25] used a ready-made PD-10 desalting column with Sephadex G-25 in water as eluent for fractionation of CDs synthesized from dextran sulfate sodium salt by the hydrothermal method and three fractions with different size were detected offline at Ve more than total column volume (Vt). Some reversible adsorption of analytes on the Sephadex G-25 gel seems to take place. The reason is likely to be the similar dextran nature of Sephadex gel and the source of the CDs mixture that was prepared. Fuyuno et al. [26] used HPSEC on three Cosmosil CNT-columns connected with online absorbance detection for fractionation of graphene CDs mixture and several different average size fractions were collected within the one broad absorption chromatographic peak, eluted after a total column volume of three columns. Typically, as the $\mathrm{CD}$ nanoparticles become smaller, the fluorescence energies are blue-shifted to higher energies [27]. However, some blue shift has been observed only in [26] and, on the contrary, the emission maxima of CD fractions increased with decreased nanoparticles size in [25] or were independent on size in [24]. The authors [24] believed that the surface state emission can play a predominant role in the fluorescence properties of CDs, leading to size-independent emission.

Thus, the efficiency of conventional SEC and HPSEC to increase the fluorescence quantum yields of CD mixtures as compared to as-prepared synthesized in various ways was shown. The SEC-separation of CD mixtures into fractions with different sizes and fluorescence properties was shown to be possible as well [24-26], but some questions about the role of adsorption of analyte on the solid phase still needs explanation. In addition, in studies discussed above SEC-experiments have been performed without detectors [20-25] or with an absorbance detector at one wavelength [26], and fractions were collected by color or by $\mathrm{V}_{\mathrm{e}}$ with subsequent offline analysis. These approaches do not allow one to be sure that the obtained fluorescent CDs are completely free of non-fluorescent components. Meanwhile, SEC coupling with online simultaneous fluorescence and absorbance measurements may be useful for application to CDs separation; however, we are not aware of any data in the literature about the isolation of CDs by this approach. Additionally, such coupling should be valuable for the understanding of CDs optical properties and could help in clarifying their nature.

In this study, the possibility of isolation of high-purity CDs fractions, free of nonfluorescent material, from natural nanographite oxide by HPSEC combined with online 
130 fluorescence and multi-wavelength absorbance measurements for the first time has been 131 shown.

132

133

134

135

136

137

138

139

140

141

142

143

144

145

146

147

148

149

150

151

152

153

154

155

156

157

158

\section{Materials and methods}

\subsection{Synthesis of NGO-MIX}

The CDs mixture was synthesized by the Hammers method from natural nanographite (NG) according to [28]. To this end, $200 \mathrm{mg}$ of $\mathrm{NG}$ was added to $50 \mathrm{ml}$ of $95 \%$ sulfuric acid (Aldrich) and 68\% nitric acid (J.T. Baker) mixture (proportion 3:1) in a $200 \mathrm{ml}$ three-neck round-bottom flask. The NG oxidation was carried out at $130{ }^{\circ} \mathrm{C}$ during $150 \mathrm{~min}$. After cooling to room temperature the supernatant was separated from insoluble graphitic residues by centrifugation at $5000 \mathrm{rpm}$ for $10 \mathrm{~min}$, diluted 10 times with distilled water, and neutralized with $1 \mathrm{M}$ sodium hydroxide (Fisher). The neutralized CDs mixture, named NGOMIX was purified from salts on Spectra/Por regenerated cellulose dialysis tubing with molecular weight cut off (MWCO) $1 \mathrm{kDa}$ for three days against distilled water and recovered by drying under vacuum at $50{ }^{\circ} \mathrm{C}$. Ten milligrams of dry NGO-MIX were dissolved in distilled water at a concentration of $2 \mathrm{mg} / \mathrm{ml}$ (optical density of about 2.2 at $270 \mathrm{~nm}$ ) and used as a stock solution for further spectroscopic and HPSEC analyses.

\subsection{Separation of fraction $N G O(3.5-10 K)$ from the bulk NGO-MIX}

Twenty milligrams of dry NGO-MIX were dissolved in $5 \mathrm{ml}$ of distilled water and size separated by centrifugation on Pall filter $10 \mathrm{~K}$ (MWCO 10kDa). Then the ultrafiltrate with nominal molecular weight $(\mathrm{NMW})<10 \mathrm{kDa}$ was dialyzed in Spectra/Por regenerated cellulose dialysis tubing with MWCO $3.5 \mathrm{kDa}$ for three days against distilled water. The resulting aquatic solution with optical density of about 1.9 at $270 \mathrm{~nm}$ containing a part of NGO-MIX with NMW between 10 and $3.5 \mathrm{kDa}$ was named fraction NGO (3.5-10K).

2.3. $U V$-visible absorption and 3D-fluorescence excitation/emission spectroscopy of NGO-MIX and fraction NGO (3.5-10K)

The UV-visible absorption spectra of aqueous NGO-MIX and its fraction NGO (3.5$10 \mathrm{~K}$ ) were recorded in a $1 \mathrm{~cm}$ quartz cuvette using a Cary 3 spectrophotometer (Varian, Cary, USA). The 3D-fluorescence excitation/emission matrix (EEM) of both samples were recorded 
159

160

161

162

163

164

165

166

167

168

169

170

171

172

173

174

175

176

177

178

179

180

181

182

183

184

185

186

187

188

189

using a Cary Eclipse fluorescence spectrophotometer (Varian, Cary, USA) in a 1-cm foursided quartz cuvette. To minimize the inner filter effect, the solutions were diluted with water to reach an absorbance of $0.05 \pm 0.01$ at $270 \mathrm{~nm}$. The excitation wavelength range was 200-520 $\mathrm{nm}$ and the emission one 250-800 nm. Stepwise increments of 10 and $1 \mathrm{~nm}$ were used for excitation and emission wavelengths, respectively.

2.4. High-performance size exclusion chromatography (HPSEC) with online fluorescence and multi-wavelength absorbance detections of NGO-MIX and fraction NGO $(3.5-10 K)$

The HPSEC of NGO-MIX and NGO (3.5-10K) was conducted on a HPLC system with multi-wavelength absorbance detector SPD-M20A (Shimadzu, Japan), operational in the 200-500 nm range. The fluorescence detector RF-20A, connected directly to the line of the absorbance detector, was set to an excitation wavelength $270 \mathrm{~nm}$, emission wavelength 450 $\mathrm{nm}$, and used for measurements of fluorescence emission. The column TSKgel G2000SW $\mathrm{XL}_{\mathrm{L}}$ (7.8 mm ID, $300 \mathrm{~mm} \mathrm{~L}$, particles size $5.0 \mu \mathrm{m}$, average pore size $12.5 \mathrm{~nm}$ ) equipped with a guard column (Tosoh Bioscience, Japan) with a fractionation range from 150 to $5 \mathrm{kDa}$ for globular proteins was used. The $30 \mathrm{mM}$ phosphate buffer ( $\mathrm{pH}$ 6.5) was used as eluent. The

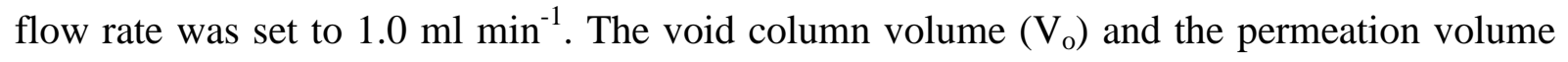
$\left(\mathrm{V}_{\mathrm{p}}\right)$ were 5.6 and $13.1 \mathrm{ml}$, respectively. The solutions of NGO-MIX and NGO (3.5-10K) applied on the column had an absorbance of 1.0 at $270 \mathrm{~nm}$ in $30 \mathrm{mM}$ phosphate buffer, and the volume of injection was $0.02 \mathrm{ml}$. The phosphate buffer used as the mobile phase did not show any absorbance or fluorescence peaks. The entire HPSEC procedure was repeated three times and the deviations did not exceed 3\%. The absorbance spectra of chromatographic peaks \#1-8 were extracted from the data of the multi-wavelength absorbance detector.

\section{Results and Discussion}

\subsection{Absorbance and 3D-fluorescence spectroscopies of NGO-MIX and fraction NGO} $(3.5-10 K)$

The bulk NGO-MIX and its NGO (3.5-10K) fraction, obtained by ultrafiltration and dialysis from the bulk sample, were subjected to UV-visible absorption and 3D-fluorescence excitation/emission analysis. The absorbance spectra of the NGO-MIX sample and fraction NGO (3.5-10K) were found to be quite different. A gradual featureless decrease of absorption 
with an increasing wavelength was observed in the NGO-MIX spectrum, while the absorption spectrum of fraction NGO (3.5-10K) had a maximum at $228 \mathrm{~nm}$ and a shoulder at $300 \mathrm{~nm}$ (Fig. 1a).

The investigation of different fluorescence centers in NGO samples was performed with excitation-emission matrix (EEM) measurements. Unexpectedly, after reviewing the scientific literature, we found that the vast majority of researchers, when studying the fluorescence properties of CDs, used a rather narrow range of excitation wavelengths, usually starting from $300 \mathrm{~nm}$ or more to $500 \mathrm{~nm}$ [20-25]. Meanwhile, the detection of new emission centers excited by UV light would be undoubtedly useful for both studying CDs structural peculiarities and their practical use. For this reason, the excitation region from 200 to $520 \mathrm{~nm}$ with a 10-nm increment was used in our EEM measurements. The EEM spectra were recorded for NGO-MIX and its fraction NGO (3.5-10K) in diluted aqueous solutions with absorbance at $270 \mathrm{~nm}$ adjusted to 0.05 to eliminate the inner filter effect and provide an emission intensity proportional to the number of emitting centers (Fig. 1). The NGO-MIX fluorescence spectra exhibited four peaks with the following excitation-emission wavelength maxima pairs $\left(\lambda_{\mathrm{exc}} / \lambda_{\mathrm{em}}\right)$ 230/440, 240/500, 290/500, and 310/435 nm/nm (Fig. 1b), while the highest emission intensity was found for the pair 230/440 nm/nm. The fraction NGO (3.5$10 \mathrm{~K}$ ) showed similar emission centers, however, the fluorescence intensity of longwavelength emitting centers $\left(\lambda_{\text {exc }} / \lambda_{\text {em }} 240 / 500\right.$ and $\left.290 / 500 \mathrm{~nm} / \mathrm{nm}\right)$ was much higher than the fluorescence intensity of short-wavelength ones $\left(\lambda_{\text {exc }} / \lambda_{\text {em }} 230 / 440\right.$ and 310/435 nm/nm) (Fig. 1d). The long-wavelength emitting centers seem to be much more concentrated in the fraction NGO (3.5-10K) than in the original NGO-MIX meaning that the essential part of fluorescence centers with short-wavelength emission was lost during the purification procedure of filtering and dialysis. In addition to local centers with high emission, both samples contained lowintensive excitation-dependent long-wavelength emitters (see Fig. 1c and 1e with conventional emission spectra extracted from EEMs). The fluorescence intensity of NGO (3.5-10K) fraction was twice or more higher than the NGO-MIX sample. Thus, the results of the absorption and 3D-fluorescence spectroscopy demonstrated that the original NGO-MIX sample and its fraction NGO (3.5-10K) are not homogeneous and can be split into individual compounds with different fluorescence properties.

\subsection{HPSEC with online fluorescence and multi-wavelength absorbance detections of} NGO-MIX and fraction NGO (3.5-10K) 
To confirm this assumption, we for the first time in the case of CDs used HPSEC with simultaneous fluorescence and multi-wavelength absorbance measurements for separation of 226 NGO-MIX and its fraction NGO (3.5-10K). Taking into account that NGO-MIX CDs nanoparticles sizes are less than $10 \mathrm{~nm}$ [28-29], we used column TSKgel G2000SW $\mathrm{XL}_{\text {with }}$ an

228 average pore size of $12.5 \mathrm{~nm}$ to achieve a satisfactory size-separating effect. The $30 \mathrm{mM}$ phosphate buffer ( $\mathrm{pH}$ 6.5) was used as eluent to suppress the possible over-exclusion effect due to the negative charge of CDs. This approach was successfully used recently for isolation of different in size fluorescent negatively charged compounds from natural dissolved organic carbon of various origins [30-31]. The excitation/emission pair 270/450 nm/nm was set for the fluorescence detector on the basis of the EEM analysis of NGO-MIX and fraction NGO (3.5-10K). At an excitation wavelength of $270 \mathrm{~nm}$ the fluorescence intensity of both samples was high and both different emission maxima in NGO-MIX well defined (Fig. 1c). The emission at $450 \mathrm{~nm}$ was used to detect all fluorophores emitting in the range 420-550 $\mathrm{nm}$ (Fig. lb,d) during one chromatographic run.

The HPSEC chromatograms of NGO-MIX and NGO (3.5-10K) are presented on Fig. 2. The UV chromatogram of NGO-MIX detected at $210 \mathrm{~nm}$ (Fig. 2 a, solid black line) showed four resolved peaks, labeled \#1, \#3, \#6, and \#8; the last one on the chromatogram's tailing edge, \#8, was considerably more intense than the other ones. On the UV chromatogram with detection at $270 \mathrm{~nm}$ (Fig. 2a, dashed black line) the intensities of peaks \#1, \#3, and \#6 were considerably lower and peak \#8 was absent. The corresponded chromatogram's fluorescence profile of NGO-MIX at $\lambda_{\text {exd }} / \lambda_{\text {em }} 270 / 450 \mathrm{~nm} / \mathrm{nm}$ (Fig. 2a, red line) showed several additional peaks, labeled \#1, \#2, \#4, \#5, and \#7. The peaks \#1-8 were eluted between the void $\left(\mathrm{V}_{\mathrm{o}} 5.6\right.$ $\mathrm{ml}$ ) and permeation $\left(\mathrm{V}_{\mathrm{p}} 13.1 \mathrm{ml}\right)$ volumes of the column (Fig. 2a) and no absorbance or fluorescence peaks after $V_{p}$ were found. This result means that fractionation was based mostly on size differences. The NGO-MIX sample was found to be heterogeneous, consisting of several size-different organic compounds, and only some of them possessed fluorescence properties. It is obvious that the main part of NGO-MIX consists of non-fluorescent compounds focused in peaks \#3, \#6, and, primarily, \#8. The fluorescent compounds (peaks $\# 1, \# 2, \# 4, \# 5, \# 7)$ seem to constitute an insignificant part of the bulk NGO-MIX sample. After HPSEC separation of NGO (3.5-10K) the UV chromatogram at $210 \mathrm{~nm}$ exhibited peaks \#1 and \#3 (Fig. 2b, solid black line). The UV chromatogram at $270 \mathrm{~nm}$ was similar in shape to that at $210 \mathrm{~nm}$ but less intense (Fig. 2b, dashed black line). The fluorescence chromatogram 
256 of NGO (3.5-10K) revealed peaks \#1 and \#4 (Fig. 2b, red line). In the NGO (3.5-10K)

257 chromatogram the absorbance intensity of peak \#3 and fluorescence intensity of peak \#4

258 increased considerably in comparison with peak \#1; peaks \#2 and \#5 were present as 259 shoulders; peaks \#6, \#7, and \#8 were absent. This result could be attributed to the 260 fractionation of NGO-MIX using ultrafiltration and dialysis. The centrifugation on the Pall 261 filter $10 \mathrm{~K}$ cut off the nanographite oxide portion with NMW more than $10 \mathrm{kDa}$, and during 262 dialysis, the compounds with NMW less than $3.5 \mathrm{kDa}$ were lost. The considerable increase of 263 the fluorescence intensity in about 5 times with the unchanged absorbance of peak \#4 in the 264 NGO (3.5-10K) chromatogram in comparison to the NGO-MIX chromatogram showed the 265 essential increase of quantum yield after purification of the NGO (3.5-10K) fraction and thus 266 the higher fluorescence purity of this material. However, in the NGO (3.5-10K) sample some 267 admixture of non-fluorescent matter located in peak \#3 was still present.

268 In the previous SEC-studies, CDs fractions differing in size were collected based on 269 their elution volumes using both visual observation [24-25] and absorbance detection at 254 $270 \mathrm{~nm}$ [26] and CDs material has been eluted as one broad peak. This approach does not allow 271 one to show reliably the presence of individual fluorescent or non-fluorescent components in 272 the CDs mixture and to accurately determine their place on the chromatographic profile. 273 Moreover, fractions were collected out of the fractionation range of the column, i.e. after the 274 total column volume [25-26]. Thus, according to the principle of SEC, it is not clear how CDs 275 fractions have been separated according to their sizes or fractions distribution were 276 additionally influenced by interactions (i.g. hydrophobic or ionic) between the gel matrix and 277 fractionated materials. On the other hand, by using our combination of HPSEC-column and 278 mobile phase with simultaneous online fluorescence and absorbance detections, the 279 fractionation of graphene CDs mixture was done based solely on size differences. For the first 280 time several individual monomodal chromatographic peaks were obtained, some of which 281 exhibited fluorescence.

282

283 284 and their HPSEC peaks

285

286

The HPSEC with a multi-wavelength absorbance detector operating in the spectral range of 287 200-500 nm allowed us to get the absorbance spectra of CDs chromophoric fractions 
288

289

290

291

292

293

294

295

296

297

298

299

300

301

302

303

304

305

306

307

308

309

310

311

312

313

314

315

316

317

318

319

09

corresponding to each chromatographic peak and to compare them with the absorbance spectra of the bulk NGO-MIX and NGO (3.5-10K) samples (Fig. 3a,b, Table 1).

The UV spectra at Ve, corresponding to peak's tops, were extracted from the multiwavelength absorbance detector data. The absorbance spectra of bulk NGO-MIX and chromatographic peak \#8 appeared to be largely similar, the contribution of other chromatographic peaks to NGO-MIX absorbance spectrum was consequential only in the region 230-320 $\mathrm{nm}$ (Fig. 2a). After normalization at $200 \mathrm{~nm}$, we found that the absorbance spectrum of peaks \#1-\#8 demonstrated different combinations of absorbance maxima (Fig. 3a-Inset, Table 1). It means that all different chromatographic peaks correspond not only to differently sized homogenous particles but to chemically different CDs fractions. The bulk NGO (3.5-10K) sample demonstrated the absorbance spectrum with the maximum at $228 \mathrm{~nm}$ and shoulder at $300 \mathrm{~nm}$ (Fig. 3b, Table 1). The shapes of absorbance spectra of peaks \#3 and \#4 were more or less similar to that of bulk NGO (3.5-10K) but had more pronounced maxima at $232 \mathrm{~nm}$ (peak \#3) and $230 \mathrm{~nm}$ (peak \#4) and different shoulders. The absorbance spectrum of peak \#1 was rather different from that of peaks \#3 and \#4, had a maximum at 221 $\mathrm{nm}$ and a shoulder at $270 \mathrm{~nm}$. We attributed the material coming with peaks \#1 and \#4 as containing different fluorescent CDs fractions. Thus, we achieved the separation of fluorescent CDs with different sizes corresponding to the material in peaks \#1, \#2, \#4, \#5, and \#7 on NGO-MIX chromatogram and in peaks \#1 and \#4 on the chromatogram of its fraction NGO (3.5-10K).

\section{Conclusions}

The NGO-MIX sample and its fraction NGO (3.5-10K) were analyzed for the first time by analytical HPSEC with a combination of online fluorescence and multi-wavelength absorbance detections. It was found that the as-prepared NGO-MIX sample was distributed in eight well-resolved CD fractions with different sizes ranged between 150 to $5 \mathrm{kDa}$ (for globular proteins) and absorption spectra, five of which were fluorescent. In NGO (3.5-10K) sample processed by ultrafiltration and dialysis, the three fractions with the lowest size were removed, decreasing the number of detected fluorescent CDs fractions but also removing the dominant non-fluorescent fraction. Despite the twice higher fluorescence intensity of fraction NGO (3.5-10K) compared to the NGO-MIX sample, some impurity of non-fluorescent components was detected in this fraction by HPSEC. The absorbance spectrum of 
chromatographic peaks, extracted from the data of multi-wavelength absorbance detector, demonstrated different combinations of absorbance maxima. It means that different chromatographic peaks correspond to sized and chemically different CDs fractions. Thus, a combination of HPSEC with simultaneous multi-wavelength absorbance and fluorescence measurements allowed the separation and identification of CDs fractions within NGO samples and monitoring of their change upon sample processing. The isolation of CDs free of nonfluorescent material will be useful for obtaining CDs with improved optical properties and sensitivity.

\section{ACKNOWLEDGMENTS}

This study has been supported by (1) Russian Foundation for Basic Research (project 19-05-00056a) in part of methodology developing and optical investigation; (2) CNRS-RAS cooperation in part of HPSEC fractionation; (3) Russian Foundation for Basic Research (project 20-32-70150) in part of preparation of NGO-MIX sample and isolation fraction NGO (3.5-10K); (4) funding in framework of state assignment. This research was performed according to the Development program of the Interdisciplinary Scientific and Educational School of Lomonosov Moscow State University «Photonic and Quantum technologies. Digital medicine».

[1] J. Fan, P.K. Chu, Group IV nanoparticles: synthesis, properties, and biological applications, Small. 6 (2010) 2080-2098. https://doi.org/10.1002/smll.201000543. [2] M. Yang, H. Li, J. Liu, W. Kong, S. Zhao, C. Li, H. Huang, Y. Liu, Z. Kang, Convenient and sensitive detection of norfloxacin with fluorescent carbon dots, J. Mater. Chem. B. 2 (2014) 7964-7970. https://doi.org/10.1039/c4tb01385a.

[3] W. Lu, X. Qin, S. Liu, G. Chang, Y. Zhang, Y. Luo, A.M. Asiri, A.O. Al-Youbi, X. Sun, Economical, green synthesis of fluorescent carbon nanoparticles and their use as probes for sensitive and selective detection of mercury(II) ions, Anal. Chem. 84 (2012) 5351-5357. https://doi.org/10.1021/ac3007939.

[4] Y. Guo, Z. Wang, H. Shao, X. Jiang, Hydrothermal synthesis of highly fluorescent carbon nanoparticles from sodium citrate and their use for the detection of mercury ions, Carbon. 52 (2013) 583-589. https://doi.org/10.1016/j.carbon.2012.10.028. 
352

353

354

355

356

357

358

359

360

361

362

363

364

365

366

367

368

369

370

371

372

373

374

375

376

377

378

379

380

381

382

383

384

[5] A. Salinas-Castillo, M. Ariza-Avidad, C. Pritz, M. Camprubí-Robles, B. Fernández, M.J. Ruedas-Rama, A. Megia-Fernández, A. Lapresta-Fernández, F. Santoyo-Gonzalez, A. Schrott-Fischer, L.F. Capitan-Vallvey, Carbon dots for copper detection with down and up conversion fluorescent properties as excitation sources, Chem. Commun. 49 (2013) 11031105. https://doi.org/10.1039/c2cc36450f.

[6] P. Miao, K. Han, Y. Tang, B. Wang, T. Lin, W. Cheng, Recent advances in carbon nanodots: synthesis, properties and biomedical applications, Nanoscale. 7 (2015) 1586-1595. https://doi.org/10.1039/c4nr05712k.

[7] N. Prabhakar, T. Näreoja, E. von Haartman, D. ŞenKaraman, S.A. Burikov, T.A. Dolenko, T. Deguchi, V. Mamaeva, P.E. Hänninen, I.I. Vlasov, O.A. Shenderova, J.M. Rosenholm, Functionalization of graphene oxide nanostructures improves photoluminescence and facilitates their use as optical probes in preclinical imaging, Nanoscale. 7 (2015) 10410 10420. https://doi.org/10.1039/c5nr01403d.

[8] O.E. Sarmanova, S.A. Burikov, S.A. Dolenko, I.V. Isaev, K.A. Laptinskiy, N. Prabhakar, D.Ş. Karaman, J.M. Rosenholm, O.A. Shenderova, T.A. Dolenko, A method for optical imaging and monitoring of the excretion of fluorescent nanocomposites from the body using artificial neural networks, Nanomedicine. $14 \quad$ (2018) 1371-1380. https://doi.org/10.1016/j.nano.2018.03.009.

[9] E. von Haartman, H. Jiang, A.A. Khomich, J. Zhang, S.A. Burikov, T.A. Dolenko, J. Ruokolainen, H. Gu, O.A. Shenderova, I.I. Vlasov, J.M. Rosenholm, Core-shell designs of photoluminescent nanodiamonds with porous silica coatings for bioimaging and drug delivery I: fabrication, J. Mater. Chem. B. 1 (2013) 2358-2366. https://doi.org/10.1039/c3tb20308e. [10] B. Yao, H. Huang, Y. Liu, Z. Kang, Carbon dots: a small conundrum, Trends Chem. 1 (2019) 235-246. https://doi.org/10.1016/j.trechm.2019.02.003.

[11] S.-T. Yang, L. Cao, P.G. Luo, F. Lu, X. Wang, H. Wang, M.J. Meziani, Y. Liu, G. Qi, Y.-P. Sun, Carbon dots for optical imaging in vivo, J. Am. Chem. Soc. 131 (2009) 1130811309. https://doi.org/10.1021/ja904843x.

[12] J. Zhong, W. Sun, Q. Wei, X. Qian, H.-M. Cheng, W. Ren, 2018. Efficient and scalable synthesis of highly aligned and compact two-dimensional nanosheet films with record performances. Nat. Commun. 9, 3484. https://doi.org/10.1038/s41467-018-05723-2.

[13] H. Li, X. He, Z. Kang, H. Huang, Y. Liu, J. Liu, S. Lian, C.H.A. Tsang, X. Yang, S.-T. Lee, Water-soluble fluorescent carbon quantum dots and photocatalyst design, Angew. Chem. Int. Ed. 49 (2010) 4430-4434. https://doi.org/10.1002/anie.200906154. 
385

386

387

388

389

390

391

392

393

394

395

396

397

398

399

400

401

402

403

404

405

406

407

408

409

410

411

412

413

414

415

[14] J. Lu, J. Yang, J. Wang, A. Lim, S. Wang, K.P. Loh, One-pot synthesis of fluorescent carbon nanoribbons, nanoparticles, and graphene by the exfoliation of graphite in ionic liquids, ACS Nano. 3 (2009) 2367-2375. https://doi.org/10.1021/nn900546b.

[15] A.A. Kokorina, A.V. Sapelkin, G.B. Sukhorukov, I.Yu. Goryacheva, 2019. Luminescent carbon nanoparticles separation and purification. Adv. Colloid Interface Sci. 274, 102043. https://doi.org/10.1016/j.cis.2019.102043.

[16] Q. Hu, X. Gong, L. Liu, M.M.F. Choi, 2017. Characterization and analytical separation of fluorescent carbon nanodots. J. Nanomater. 2017, 1804178. https://doi.org/10.1155/2017/1804178.

[17] Y. Lu, J. Wang, H. Yuan, D. Xiao, Separation of carbon quantum dots on a C18 column by binary gradient elution via HPLC, Anal. Methods. 20 (2014) 8124-8128. https://doi.org/ 10.1039/c4ay01052c.

[18] Q. Hu, M. Chin Paau, Y. Zhang, W. Chan, X. Gong, L. Zhang, M.M.F. Choi, Capillary electrophoretic study of amine/carboxylic acid-functionalized carbon nanodots. J. Chromatogr. A. 1304 (2013) 234-240. https://doi.org/10.1016/j.chroma.2013.07.035.

[19] Q. Wu, X. Zhang, X. Zhang, S. Dong, H. Qiu, L. Wang, Multi-mode application of graphene quantum dots bonded silica stationary phase for high performance liquid chromatography. J. Chromatogr. A. $1492 \quad$ (2017) 61-69. https://doi.org/10.1016/j.chroma.2017.02.067.

[20] H. Zheng, Q. Wang, Y. Long, H. Zhang, X. Huang, R. Zhu, Enhancing the luminescence of carbon dots with a reduction pathway, Chem. Commun. 47 (2011) 10650-10652. https://doi.org/10.1039/c1cc14741b.

[21] X. Wang, L. Cao, S.-T. Yang, F. Lu, M.J. Meziani, L. Tian, K.W. Sun, M.A. Bloodgood, Y.-P. Sun, Bandgap-like strong fluorescence in functionalized carbon nanoparticles, Angew. Chem. Int. Ed. 49 (2010) 5310-5314. https://doi.org/10.1002/anie.201000982.

[22] P. Anilkumar, X. Wang, L. Cao, S. Sahu, J.-H. Liu, P. Wang, K. Korch, K.N. Tackett II, A. Parenzan and Y.-P. Sun, Toward quantitatively fluorescent carbon-based ' quantum' dots. Nanoscale. 3 (2011) 2023-2027.

[23] C. Jiang, H. Wu, X. Song, X. Ma, J. Wang, M. Tan, Presence of photoluminescent carbon dots in Nescafe® original instant coffee: applications to bioimaging, Talanta. 127 (2014) 68-74. https://doi.org/10.1016/j.talanta.2014.01.046. 
416 [24] F. Arcudi, L. Đorđević, M. Prato, Synthesis, separation, and characterization of small and 417 highly fluorescent nitrogen-doped carbon nanodots, Angew. Chem. Int. Ed. 55 (2016) 2107418 2112. https://doi.org/10.1002/anie.201510158.

419 [25] A.A. Kokorina, E.S. Prikhozhdenko, N.V. Tarakina, A.V. Sapelkin, G.B. Sukhorukov, 420 I.Y. Goryacheva, Dispersion of optical and structural properties in gel column separated 421 carbon nanoparticles, $\quad$ Carbon. $127 \quad$ 541-547. 422 https://doi.org/10.1016/j.carbon.2017.11.039.

423 [26] N. Fuyuno, D. Kozawa, Y. Miyauchi, S. Mouri, R. Kitaura, H. Shinohara, T. Yasuda, N. 424 Komatsu, K. Matsuda, Drastic change in photoluminescence properties of graphene quantum 425 dots by chromatographic separation. Adv. Opt. Mater. 2 (2014) 983-989. 426 https://doi.org/10.1002/adom.201400200.

427 [27] R. Ye, C. Xiang, J. Lin, Z. Peng, K. Huang, Z. Yan, N.P. Cook, E.L.G. Samuel, C.-C. 428 Hwang, G. Ruan, G. Ceriotti, A.-R.O. Raji, A.A. Marti, J.M. Tour, 2013. Coal as an abundant 429 source of graphene quantum dots. Nat. Commun. 4, 2943. 430 https://doi.org/10.1038/ncomms3943.

431 [28] S. Ciftan Hens, W.G. Lawrence, A.S. Kumbhar, O. Shenderova, Photoluminescent 432 nanostructures from graphite oxidation, J. Phys. Chem. C. 116 (2012) 20015-20022. 433 https://doi.org/10.1021/jp303061e.

434 [29] K.A. Laptinskiy, S.A. Burikov, S.V. Patsaeva, I.I. Vlasov, O.A. Shenderova, T.A. 435 Dolenko, 2020. Absolute luminescence quantum yield for nanosized carbon particles in water 436 as a function of excitation wavelength. Spectrochim. Acta A. 229, 117879. 437 https://doi.org/10.1016/j.saa.2019.117879.

438 [30] O.E. Trubetskaya, C. Richard, S.V. Patsaeva, O.A. Trubetskoj, 2020. Evaluation of 439 aliphatic/aromatic compounds and fluorophores in dissolved organic matter of contrasting 440 natural waters by SEC-HPLC with multi-wavelength absorbance and fluorescence detections. 441 Spectrochim. Acta A. 238, 118450. https://doi.org/10.1016/j.saa.2020.118450.

442 [31] O.A. Trubetskoj, C. Richard, G. Voyard, V.V. Marchenkov, O.E. Trubetskaya, 443 Molecular size distribution of fluorophores in aquatic natural organic matter: application of 444 HPSEC with multi-wavelength absorption and fluorescence detection following LPSEC445 PAGE fractionation, Environ. Sci. Technol. $52 \quad$ (2018) 5287-5295. 446 https://doi.org/10.1021/acs.est.7b03924. 

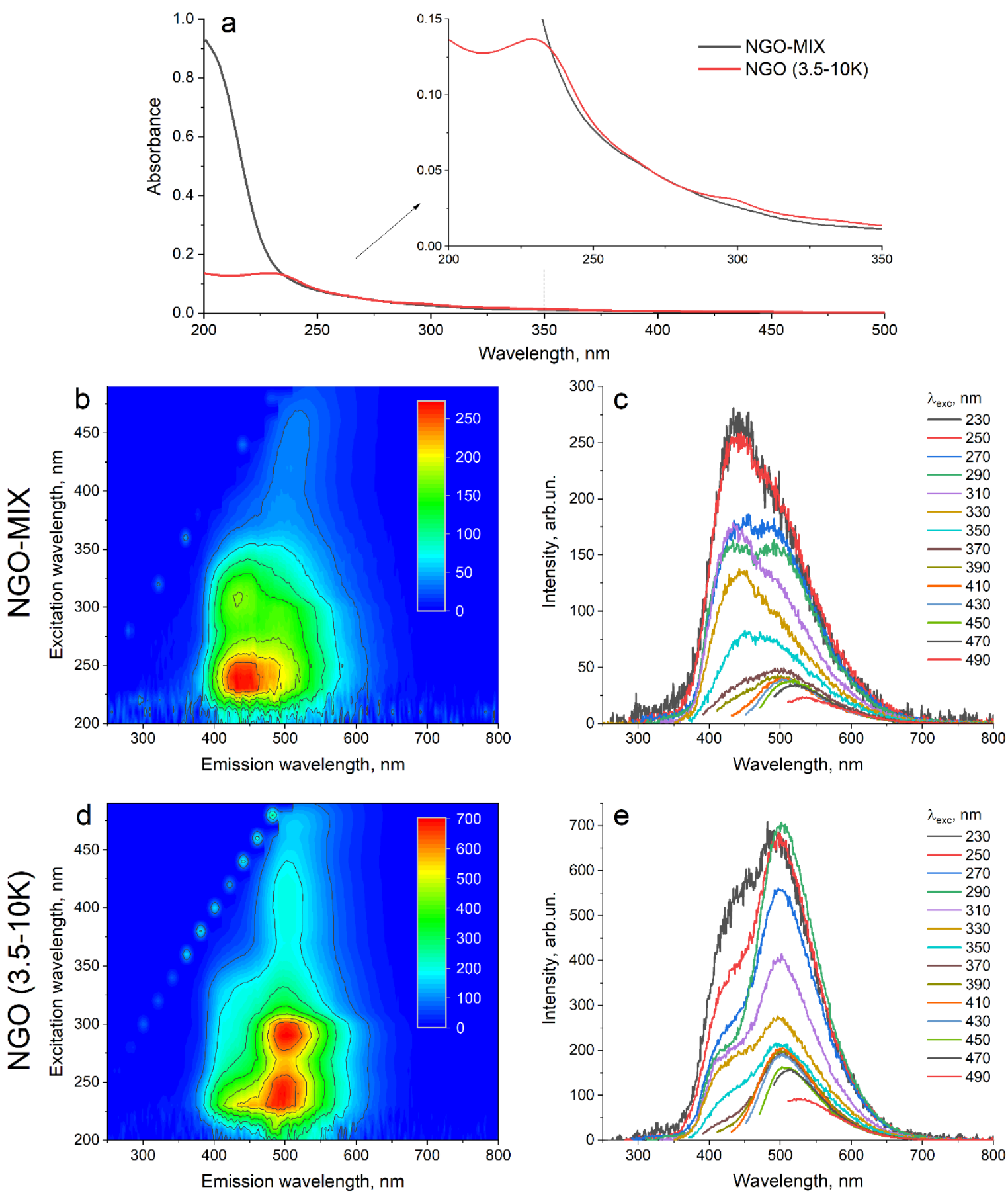

448 Figure 1. The absorbance spectra of NGO-MIX and NGO (3.5-10K) samples (a). The EEM 449 of NGO-MIX (b) and NGO (3.5-10K) (d), the sets of conventional emission spectra of NGO450 MIX (c) and NGO (3.5-10K) (e). The absorbance and fluorescence spectra were recorded for 451 NGO aqueous solutions with absorption of 0.05 at $270 \mathrm{~nm}$. 
453
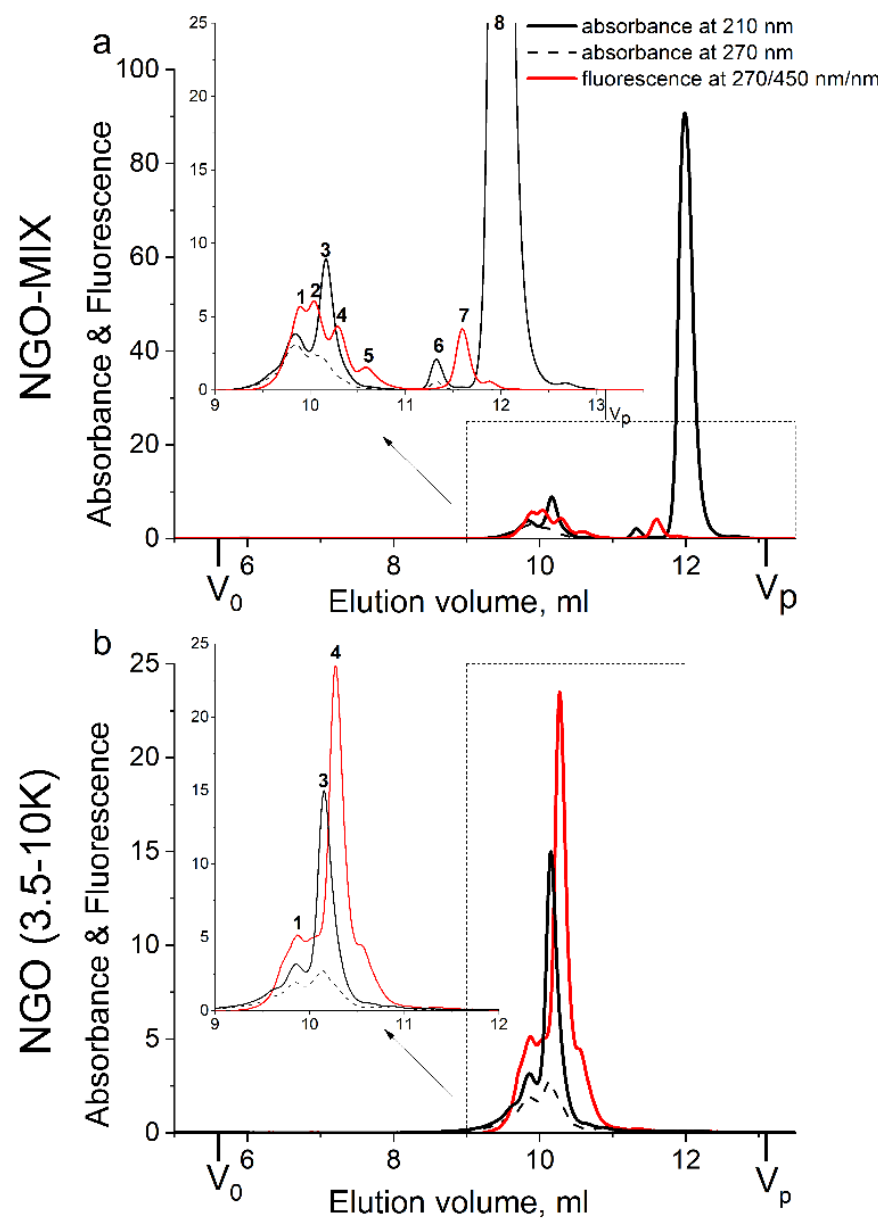

454 Figure 2. HPSEC chromatograms of NGO-MIX (a) and NGO (3.5-10K) (b) on TSKgel 455 G2000SW $\mathrm{XL}_{\mathrm{X}}$ column in $30 \mathrm{mM}$ phosphate buffer (pH 6.5) with absorbance detections (at 210 $456 \mathrm{~nm}$, solid black lines, and at $270 \mathrm{~nm}$, dashed black lines) and fluorescence detection (at $457 \lambda_{\text {exc }} / \lambda_{\text {em }}-270 / 450 \mathrm{~nm} / \mathrm{nm}$, solid red lines). 

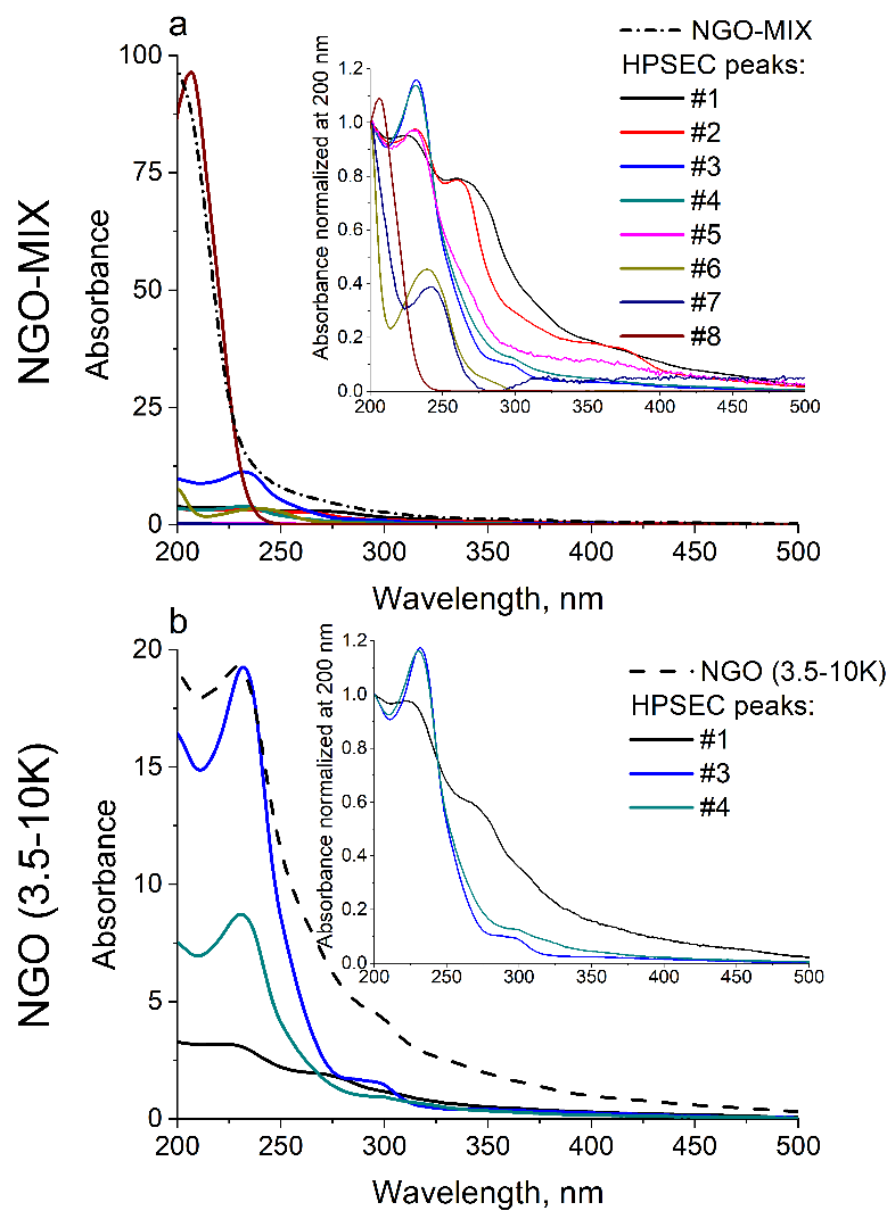

459

460

Figure 3. Absorbance spectra of NGO-MIX and its HPSEC peaks \#1-\#8 (a), of NGO (3.5-

461 10K) and its HPSEC peaks \#1, \#3, and \#4 (b). Insets: normalized at $200 \mathrm{~nm}$ absorbance 462 spectra of NGO-MIX peaks \#1-\#8 (a) and NGO (3.5-10K) peaks \#1, \#3, and \#4 (b). 
464 Table 1. Elution volumes $\left(\mathrm{V}_{\mathrm{e}}\right)$ and absorbance maxima in UV-vis region of the bulk NGO465 MIX, NGO (3.5-10K) and HPSEC peaks. The absorbance spectra were extracted from the 466 data of the multi-wavelength absorbance detector at $\mathrm{V}_{\mathrm{e}}$ corresponded to the chromatographic 467 peaks' tops.

\begin{tabular}{|c|c|c|c|}
\hline \multirow{2}{*}{ Peak number } & \multirow{2}{*}{$\mathrm{V}_{\mathrm{e}}, \mathrm{ml}$} & \multicolumn{2}{|c|}{ Absorbance maxima, nm } \\
\hline & & NGO-MIX & NGO $(3.5-10 \mathrm{~K})$ \\
\hline Bulk sample & - & No max & $\begin{array}{c}228 \\
\text { sh*300 }\end{array}$ \\
\hline 1 & 9.89 & $\begin{array}{l}224 \\
260\end{array}$ & $\begin{array}{c}221 \\
\operatorname{sh} 270\end{array}$ \\
\hline 2 & 10.03 & $\begin{array}{c}231 \\
260 \\
\text { sh } 365\end{array}$ & - \\
\hline 3 & 10.15 & $\begin{array}{c}232 \\
\text { sh } 290 \\
\text { sh } 365\end{array}$ & $\begin{array}{c}232 \\
\operatorname{sh} 290\end{array}$ \\
\hline 4 & 10.29 & $\begin{array}{c}230 \\
\text { sh } 300\end{array}$ & $\begin{array}{c}230 \\
\operatorname{sh} 300\end{array}$ \\
\hline 5 & 10.59 & $\begin{array}{c}230 \\
\text { sh } 260 \\
\text { sh } 350\end{array}$ & - \\
\hline 6 & 11.31 & $\begin{array}{c}238 \\
\operatorname{sh} 280\end{array}$ & - \\
\hline 7 & 11.59 & $\begin{array}{l}242 \\
320\end{array}$ & - \\
\hline 8 & 12.00 & 206 & - \\
\hline
\end{tabular}

*sh-shoulder 